\title{
Dynamics of vibrations in a mixed amorphous-nanocrystalline Si system
}

\author{
Marjolein van der Voort, ${ }^{1}$ Otto L. Muskens, ${ }^{1}$ Andrey V. Akimov, ${ }^{1,2}$ Alexander B. Pevtsov, ${ }^{2}$ and Jaap I. Dijkhuis ${ }^{1}$ \\ ${ }^{1}$ Faculty of Physics and Astronomy, Debye Institute, Utrecht University, P.O. Box 80.000, 3508 TA Utrecht, The Netherlands \\ ${ }^{2}$ A.F.Ioffe Physical-Technical Institute, Russian Academy of Sciences, 194021 St. Petersburg, Russia
}

(Received 1 December 2000; published 21 June 2001)

\begin{abstract}
We present results of pulsed Raman and phonon-induced luminescence experiments on a mixed amorphousnanocrystalline silicon system $(a-\mathrm{nc}-\mathrm{Si}: \mathrm{H})$. With these experiments, the decay and transport of nonequilibrium phonons in $a$-nc-Si:H was examined and compared with the behavior of phonons of the same frequencies in $a$-Si:H and $c$-Si. From the Raman measurements, we find that in the spectral region of the TO vibrations in the crystallites $\left(505-520 \mathrm{~cm}^{-1}\right)$, phonons have shorter decay times than the TO phonons in $a-\mathrm{Si}: \mathrm{H}$, but longer than in $c$-Si. In addition, the lifetimes increase with decreasing frequency, from less than $10 \mathrm{~ns}$ at $515 \mathrm{~cm}^{-1}$ to $\sim 30 \mathrm{~ns}$ at $505 \mathrm{~cm}^{-1}$. We further show that phonons with a frequency of $\sim 150 \mathrm{~cm}^{-1}$ in $a$-nc-Si:H have longer lifetimes than in $a-\mathrm{Si}: \mathrm{H} \quad(\tau<10 \mathrm{~ns})$. Finally, the diffusion of $29-\mathrm{cm}^{-1}$ phonons through the $a-\mathrm{nc}-\mathrm{Si}: \mathrm{H}$ and $a-\mathrm{Si}: \mathrm{H}$ material was examined in phonon-induced luminescence experiments. Transport through the $a$-nc-Si:H film appears to be much slower than through the $a$-Si:H layer. We explain these results as effects of phonon confinement, and relate them to the extremely long phonon lifetimes found in Raman experiments on $a-\mathrm{Si}(: \mathrm{H})$.
\end{abstract}

DOI: 10.1103/PhysRevB.64.045203

PACS number(s): 63.22. $+\mathrm{m}, 63.50 .+\mathrm{x}$

\section{INTRODUCTION}

Numerous studies have been carried out to investigate the effects of confinement on the properties of phonons in semiconductor nanoparticles. ${ }^{1}$ In Raman scattering experiments, size-dependent shifts and broadening of the peaks of optical and acoustic phonons have been observed. ${ }^{2-4}$ Yet only a few examples exist of experiments in which the dynamical aspects of confined phonons have been studied. In porous nanocrystalline corundum, one has measured millisecond lifetimes of size-quantized vibrations with a frequency of $20 \mathrm{~cm}^{-1}$. In small-grain polycrystalline corundum the decay of phonons with frequencies between 29 and $80 \mathrm{~cm}^{-1}$ has been examined. Also in those experiments, surprisingly long phonon decay times ( $\tau>1 \mathrm{~ms}$ ) have been discovered. ${ }^{6}$ In the corundum-based materials, the vibrational dynamics turned out to be rather sensitive to the ratio of the acoustic wavelength $\lambda$ of the vibrational excitation studied and the typical size $a$ of the microstructures that constitute the system. For $\lambda / a \ll 1$, the observed phonon dynamics is similar to that encountered in bulk crystals: the measured phonon lifetimes are of the same order of magnitude, and the phonon propagation appeared to be almost ballistic. ${ }^{7}$ In contrast, for $\lambda / a \sim 1$, vibrational dynamics resemble more the behavior observed in glasses: the vibrations decay on much longer time scales, and propagate diffusively. ${ }^{8,9}$ The transition from "crystalline" to "glassy" dynamics appeared to be gradual.

These observations have inspired us to examine to what extent the anomalous vibrational properties of glassy and amorphous solids are related to their microstructure. Scholten et al. investigated the dynamics of high-frequency phonons in (hydrogenated) amorphous silicon. ${ }^{8,10,11}$ They measured phonon lifetimes more than three orders of magnitude longer than the lifetimes of phonons of the same energy in $c$-Si. Furthermore, lifetimes appeared to increase with increasing frequency, as opposed to the frequency dependence generally encountered in crystals. In contrast, calculations of anharmonic decay rates in numerically built models of $a-\mathrm{Si}$ have predicted picosecond lifetimes for the same highfrequency modes, and a decrease of the vibrational lifetimes with increasing frequency. ${ }^{12}$ These remarkable contradictions could be related to the microstructure of real $a-\mathrm{Si}(: \mathrm{H})$. In the computer simulations, $a-\mathrm{Si}$ is assumed to be statistically homogeneous. However, it has been proposed that $a$-Si $(: \mathrm{H})$ consists of small $(\sim 1 \mathrm{~nm})$ building blocks, ${ }^{13,14}$ and contains numerous internal microscopic surfaces. The presence of these may result in phonon interference effects, and, if the blocks are decoupled from the surrounding, in a suppression of the anharmonic decay rates.

To justify such ideas, it is of interest to study the dynamics of vibrations in a mixed amorphous-nanocrystalline material, which exhibits crystalline order on nanometer length scales. Such experiments may provide information contributing to the understanding of the dynamics of phonons in amorphous systems.

In this paper we present results of pulsed Raman and phonon-induced luminescence experiments on a mixed amorphous-nanocrystalline silicon system $(a$-nc-Si:H). With these techniques we examined the decay of high-frequency phonons $\left(\sim 150-530 \mathrm{~cm}^{-1}\right)$, and transport of phonons with a frequency $\left(\sim 29 \mathrm{~cm}^{-1}\right)$ close to the expected lowest frequency supported by the nanoparticles in $a$-nc-Si:H. Section II describes the experimental techniques used. The results of the measurements are presented in Sec. III. In Sec. IV we analyze the observations and discuss the influence of the materials microstructure on the phonon dynamics. The results are compared with the behavior of phonons of the same frequencies in $a$-Si:H. In Sec. V we summarize and conclude. For convenience, throughout the paper the vibrational excitations are called "phonons."

\section{EXPERIMENTAL DETAILS}

To examine the difference between phonon dynamics in pure $a$-Si:H and mixed amorphous-nanocrystalline $\mathrm{Si}$ 
( $a$-nc-Si:H) layers, two types of samples were prepared. Both were grown on sapphire $\left(\mathrm{Al}_{2} \mathrm{O}_{3}\right)$ wafers, by means of plasma-enhanced chemical vapor deposition, to a thickness of $\sim 0.4 \mu \mathrm{m}$. The substrates contained about 20 at. ppm of $\mathrm{Cr}^{3+}$ ions. By carefully controlling the deposition parameters, an $a$-Si:H and an $a$-nc-Si:H film were produced during the same run. The $a$-nc-Si:H layer was made to contain a large volume fraction $(\sim 24 \%)$ of $\mathrm{Si}$ crystallites with an average diameter of $\sim 4.5 \mathrm{~nm}$, as was estimated from a careful analysis of the Raman spectra and electrical conductivity of the film. ${ }^{15}$ High-resolution electron microscopy studies have further shown a dispersion in crystallite sizes. The smallest particles observed have diameters of about $1.5 \mathrm{~nm}$. For details on the sample preparation, we refer to Ref. 15.

All experiments were carried out on samples held at a temperature of $1.8 \mathrm{~K}$, in order to reduce the thermal phonon population in the energy range of interest. A nonequilibrium vibrational population was created during the fast relaxation and recombination of optically excited charge carriers.

Two types of experiments were performed. By means of time-resolved Raman spectroscopy, the decay of phonons with a frequency between $\sim 150$ and $530 \mathrm{~cm}^{-1}$ was examined. Phonon-induced luminescence experiments were carried out to investigate the transport of $29-\mathrm{cm}^{-1}$ phonons through the $a-\mathrm{Si}: \mathrm{H}$ and $a$-nc-Si:H layers.

\section{A. Time-resolved Raman spectroscopy}

An effective method to investigate the dynamics of highfrequency vibrations in amorphous semiconductors is provided by pulsed Raman spectroscopy. Scholten et al. ${ }^{8,10}$ employed this technique to examine transient phonon populations in $a$-Si:(H). Since the room-temperature Raman spectra of our $a$-nc-Si:H samples are quite similar to those of $a-\mathrm{Si}: \mathrm{H}$, we expected that pulsed Raman methods could also be used to study the temporal evolution of phonon populations in $a$-nc-Si:H. In addition, it is known that in roomtemperature Stokes Raman spectra of $a$-nc-Si:H, the contributions of phonons in $a-\mathrm{Si}: \mathrm{H}$ and the nanocrystallites can be distinguished in the frequency range of the TO phonons. The Stokes TO peak of our samples consists of a superposition of a broad amorphous TO peak (centered at $480 \mathrm{~cm}^{-1}$ ) and a $15-\mathrm{cm}^{-1}$-wide line centered at $515 \mathrm{~cm}^{-1}$ that corresponds to TO vibrations in the crystallites (see Fig. 1). One of the goals of the Raman measurements was to see whether differences can be observed between the dynamics of TO phonons in $a$-Si:H and the crystallites.

In the Raman experiments described below, phonon generation was accomplished with the output of two $Q$-switched, frequency-doubled Nd:YAG (yttrium aluminum garnet) lasers $(\lambda=532 \mathrm{~nm}$, penetration depth into the samples of $\sim 100 \mathrm{~nm}$ ). Both lasers generated a train of 10-ns pulses with a repetition rate of $30 \mathrm{~Hz}$. The beams were focused to a $\sim 1-\mathrm{mm}^{2}$ spot on the sample, where the average power of each beam typically amounted to $10 \mathrm{~mW}$, corresponding to an absorbed energy density per pulse of $\sim 3 \times 10^{3} \mathrm{~J} / \mathrm{cm}^{3}$. To examine the temporal evolution of the phonon populations created, pulses of one of the two lasers (probe) were given an electronically controlled delay with respect to those of the

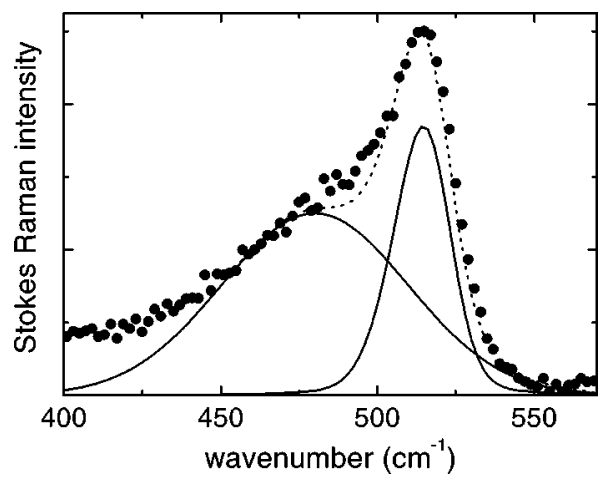

FIG. 1. Room-temperature Stokes Raman spectrum of the $a$-nc-Si:H sample in the frequency range of the TO phonons. The solid lines indicate the Raman contributions from $a-\mathrm{Si}: \mathrm{H}$ and $\mathrm{nc}-\mathrm{Si}$, centered at $480 \mathrm{~cm}^{-1}$ and $515 \mathrm{~cm}^{-1}$, respectively, and the dotted line is their sum. The spectrum was excited with an argon-ion laser.

other (pump). By measuring anti-Stokes intensities as a function of the delay between pump and probe, the vibrational population decay was monitored on time scales of $10 \mathrm{~ns}$ up to $15 \mathrm{~ms}$.

In the first series of experiments, the Raman scattered light was analyzed by a triple monochromator, and detected by a charged-coupled device (CCD) camera, with a 15-min exposure time. In this way, anti-Stokes spectra were recorded with a spectral resolution of $\sim 20 \mathrm{~cm}^{-1}$.

A second series of measurements was carried out with a higher spectral resolution in order to study the dynamics of TO phonons as a function of the frequency within the TO peak. These experiments were performed to search for differences between the behavior of TO phonons in $a-\mathrm{Si}: \mathrm{H}$ and the crystallites. Therefore, the experimental setup was modified. A larger, conventional double monochromator was used, equipped with a Peltier-cooled photomultiplier tube, followed by photon-counting electronics. A time-toamplitude converter was used to define a 500-ns gate. Only photons detected during the selected interval were recorded, so that the majority of dark counts was rejected. In contrast with the CCD setup, Raman spectra were obtained by recording time traces for different spectral positions of the monochromator. The decay of the phonon population was studied by performing the experiments as a function of the delay between the pump and probe pulses. Stokes measurements were averaged over $500 \mathrm{~s}$, anti-Stokes signals over $1500 \mathrm{~s}$. The spectral slit width of the double monochromator amounted to $12 \mathrm{~cm}^{-1}$. All Raman measurements were performed on samples immersed in superfluid He, at a temperature of $\sim 1.8 \mathrm{~K}$.

\section{B. Phonon-induced luminescence experiments}

To extend our investigations to a larger frequency range than the $150-530-\mathrm{cm}^{-1}$ range addressed by the Raman measurements, phonon-induced luminescence experiments were performed. Advantage was taken of the fact that the layers studied were grown on sapphire, which in fact is very dilute ruby, and can be used as an optical detector for $29-\mathrm{cm}^{-1}$ phonons injected by the $a-\mathrm{nc}-\mathrm{Si}: \mathrm{H}$ and $a-\mathrm{Si}: \mathrm{H}$ films into the 
substrate. ${ }^{11}$ A study of the transport of $29-\mathrm{cm}^{-1}$ phonons through the $a$-nc-Si:H layer is of particular interest, as the frequency of these phonons is of the order of the lowest frequency that can be supported by the nanometer-size crystallites present in the $a$-nc-Si:H film. Under such conditions, the materials microstructure may have pronounced effects on the phonon dynamics.

The principle of the ruby phonon detector is based on the energy levels of the $\mathrm{Cr}^{3+}$ ions present in the substrate, and the radiative and nonradiative transitions between them. ${ }^{16}$ In the absence of $29-\mathrm{cm}^{-1}$ phonons, optical excitation of $\mathrm{Cr}^{3+}$ results in red luminescence corresponding to the radiative transition from the $\bar{E}\left({ }^{2} E\right)$ level to the ground state $\left(R_{1}\right)$. When $29-\mathrm{cm}^{-1}$ phonons are injected into the excited sapphire, transitions from the $\bar{E}\left({ }^{2} E\right)$ to the $2 \bar{A}\left({ }^{2} E\right)$ state take place, by resonant absorption of the $29-\mathrm{cm}^{-1}$ phonons. This process is followed by radiative decay from the $2 \bar{A}\left({ }^{2} E\right)$ to the ground state $\left(R_{2}\right.$ luminescence). The temporal evolution of the $R_{2}$ intensity directly reflects the flux of $29-\mathrm{cm}^{-1}$ phonons from the sample into the sapphire substrate. The ratio of $R_{2} / R_{1}$ is proportional to the $29-\mathrm{cm}^{-1}$ phonon occupation number in the sample. For details on the workings of the ruby detector and its application in extensive studies of the transport of $29-\mathrm{cm}^{-1}$ phonons in $a-\mathrm{Si}: \mathrm{H}$, we refer to Ref. 11.

Also in the ruby experiments, phonons were generated in the samples during the relaxation and recombination of hot carriers excited by the absorption of green photons. The green light $(\lambda=514.5 \mathrm{~nm}$, penetration depth into $a-\mathrm{Si}: \mathrm{H}$ $\sim 100 \mathrm{~nm}$ ) was emitted by a mode-locked, cavity-dumped argon-ion laser producing 1-ns pulses at repetition rates of $800 \mathrm{kHz}$ or $4 \mathrm{MHz}$. The beam was focused to a $\sim 100$ - $\mu \mathrm{m}$-diam spot on the sample, where the average excitation power amounted to $\sim 4 \mathrm{~mW}$. For a repetition rate of $4 \mathrm{MHz}$ this corresponds to an absorbed energy density of $\sim 1.3 \mathrm{~J} / \mathrm{cm}^{3}$ per pulse. Part of the light that was not absorbed by the $a(-n c)-S i: H$ layers excited $\mathrm{Cr}^{3+}$ ions in the substrate, and activated the ruby phonon detector. Luminescence from the substrate was collected and projected on the entrance slit of a double monochromator with a spectral slit width set to $\sim 5 \mathrm{~cm}^{-1}$. The red photons were detected by means of a Peltier-cooled photomultiplier tube, followed by photon-counting electronics. The temporal evolution of the $29-\mathrm{cm}^{-1}$ phonon population emitted by the sample was monitored with a resolution of $\sim 10 \mathrm{~ns}$, limited by the speed of the ruby phonon detector, as discussed in (Ref. 11). The samples were cooled with $\mathrm{He}$ gas, in a continuous-flow cryostat operating at $1.8 \mathrm{~K}$.

\section{EXPERIMENTAL RESULTS}

\section{A. Raman experiments}

Figure 2 shows low-temperature Stokes and anti-Stokes Raman spectra of both the $a$-Si:H and $a$-nc-Si:H samples, obtained with the CCD detection scheme. The broad lines typical for TA-, LA-, and TO-like phonons in $a-\mathrm{Si}: \mathrm{H}$ can be distinguished. From Fig. 1 we know that the Stokes TO peak of the $a$-nc-Si:H layer consists of a superposition of a broad

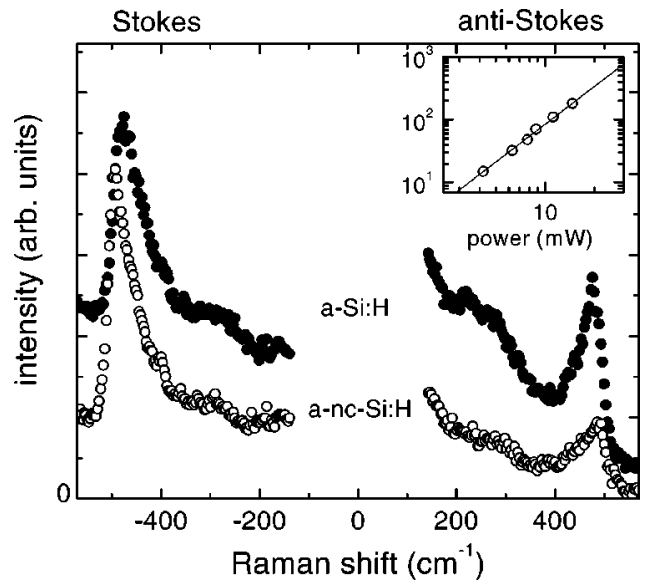

FIG. 2. Raman spectra of $a$-nc-Si:H (O) and $a$-Si:H ( $(\bullet)$ as recorded with the $\mathrm{CCD}$ for $T \sim 1.8 \mathrm{~K}$. Anti-Stokes intensities have been multiplied by a factor of 2.5. The inset shows the dependence of the TO anti-Stokes signal of the $a$-nc-Si:H sample on excitation power for excitation with one laser. The line indicates a quadratic dependence on power.

amorphous TO peak and a narrower line centered at $515 \mathrm{~cm}^{-1}$ that corresponds to TO phonons in nc-Si. However, in case of the measurements presented in Fig. 2, the resolution of the spectrometer was not sufficient to resolve the amorphous and nanocrystallite peaks: only a shift of the total TO peak is observed. Later in this section, lowtemperature spectra will be presented that were taken with a higher spectral resolution.

Next, we examined for the $a$-nc-Si:H sample the dependence of the Raman intensity on excitation power $P$. As in $a$-Si $(: \mathrm{H}),{ }^{8}$ the Stokes intensities $I_{\mathrm{S}}(\omega)$ appeared to increase linearly with $P$, whereas the anti-Stokes signals $I_{\mathrm{AS}}(\omega)$ exhibit a quadratic dependence on excitation power. The inset of Fig. 2 illustrates the $P^{2}$ dependence of the anti-Stokes TO intensity of $a$-nc-Si:H. Phonon occupation numbers $n(\omega)$ can be derived from the well-known relation $n(\omega)$ $=I_{\mathrm{AS}}(\omega) /\left[I_{\mathrm{S}}(\omega)-I_{\mathrm{AS}}(\omega)\right]$. For the frequencies and powers presented, $n(\omega)$ ranged between $\sim 0.03$ and 0.3 , so that $n(\omega) \approx I_{\mathrm{AS}}(\omega) / I_{\mathrm{S}}(\omega)$. Then, the observed dependencies of $I_{\mathrm{AS}}$ and $I_{\mathrm{S}}$ on excitation power imply that $n(\omega)$ increases linearly with $P$, independent of $\omega$. This demonstrates that the phonon distribution studied is not thermalized and has a nonequilibrium character. ${ }^{8}$

The temporal evolution of the generated nonequilibrium phonon population was investigated by recording a series of anti-Stokes spectra for different delays $\Delta t$ between the pulses of the pump and probe laser. To extract the timedependent contribution to the spectra, a spectrum taken with a delay of $15 \mathrm{~ms}$ was subtracted from each measurement. The intensity of the $\Delta t=15 \mathrm{~ms}$ spectrum, namely, is equal to the sum of the separate contributions of the two lasers and hence could be used as a background spectrum. Also a 30\% background corresponding to electronic effects occurring in both plasma-enhanced chemical-vapor deposition-grown $a$-Si:H and $a$-nc-Si:H (Ref. 17) was subtracted from the signals, to focus the attention on the differences in phonon dynamics in the two systems. 


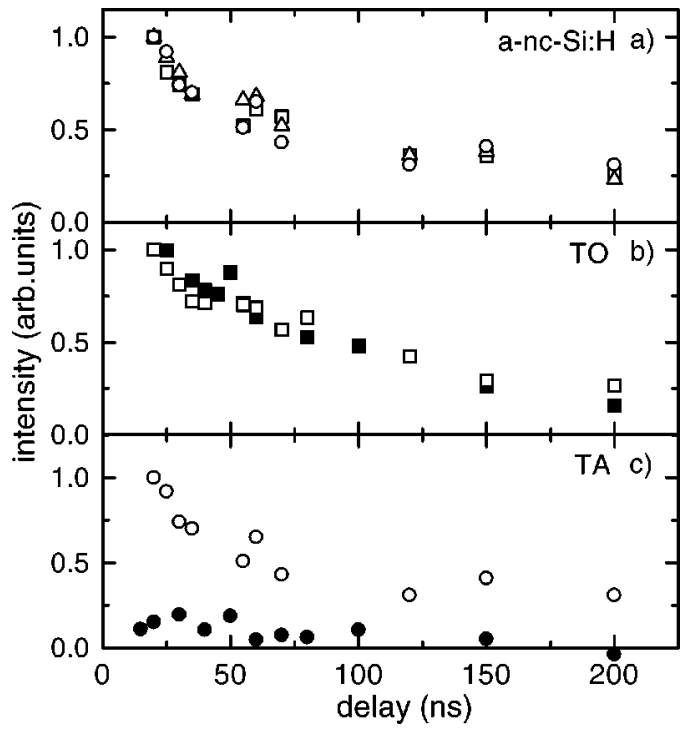

FIG. 3. (a) Normalized anti-Stokes intensities as a function of the delay, measured in $a$-nc-Si:H in the spectral regions of TO ( $\square$ ), LA $(\triangle)$, and TA $(\bigcirc)$ vibrations. (b) Normalized TO anti-Stokes intensities vs delay in $a$-nc-Si:H ( $\square$ ) and $a-\mathrm{Si}: \mathrm{H}$ (ם). (c) Normalized TA anti-Stokes intensities vs delay in $a$-nc-Si:H $(\bigcirc)$ and $a-\mathrm{Si}: \mathrm{H}(\bigcirc)$. To extract the time-dependent contributions to $I_{\mathrm{AS}}(\omega)$, spectra taken with a delay of $15 \mathrm{~ms}$ and a $30 \%$ background corresponding to electronic effects (Ref. 17) were subtracted from all signals.

In Fig. 3 we present the results as normalized anti-Stokes Raman intensities plotted versus $\Delta t$. Figure 3(a) shows the temporal evolution of $I_{\mathrm{AS}}(\omega)$ in the $a$-nc-Si:H layer, for three spectral regions. To improve signal-to-noise ratios, all intensities were integrated over a range of frequencies. For the TA, LA, and TO phonons, the integration was carried out over a range of $140-220 \mathrm{~cm}^{-1}, 240-380 \mathrm{~cm}^{-1}$, and $420-$ $550 \mathrm{~cm}^{-1}$, respectively. In Fig. 3(b), the behavior of TO phonons in $a$-Si:H and $a$-nc-Si:H is compared, and Fig. 3(c) shows how the temporal evolution of $I_{\mathrm{AS}}$ for the frequency region of the TA phonons differs in the two materials.

As can be seen, the decay of the TA, LA, and TO phonon population in $a$-nc-Si:H closely resembles the decay of the TO phonons in $a-\mathrm{Si}: \mathrm{H}$ without nanocrystals, both with respect to the temporal shape of the signals as to the absolute values of the decay times. Therefore, the most pronounced difference in vibrational dynamics we observe from these measurements concerns the behavior of the TA phonons. Figure 3(c) shows that the TA population in $a-\mathrm{Si}: \mathrm{H}$ decays faster than can be resolved with the 10-ns resolution of the experimental setup, which is not the case for phonons of the same frequency in $a$-nc-Si:H. So even if the signals for the TA frequencies reflect the decay of higher-frequency vibrations that feed the TA population, the TA lifetimes in $a$-nc-Si:H have to be significantly longer than in $a-\mathrm{Si}: \mathrm{H}$. We note that the result for $a-\mathrm{Si}: \mathrm{H}$ is consistent with the nanosecond lifetimes reported for TA's in $a-\mathrm{Si}(: \mathrm{H})$ by Scholten et al. ${ }^{8,10}$

A second series of measurements was performed with the higher spectral resolution of the modified Raman setup with

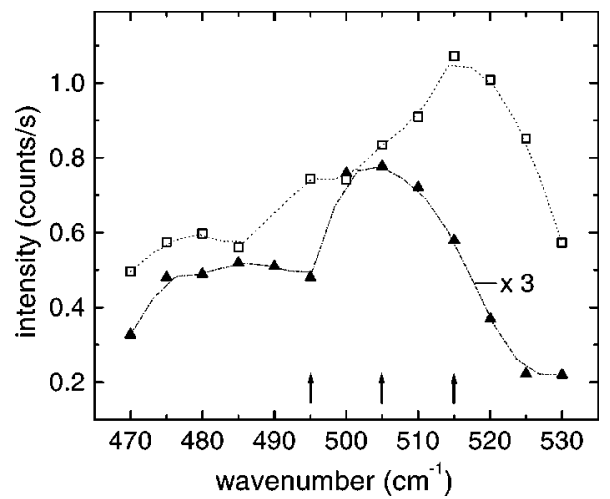

FIG. 4. Stokes $(\square)$ and anti-Stokes ( $\boldsymbol{\Delta})$ TO peaks of the $a$-nc-Si:H film, obtained with the gated detection scheme. AntiStokes intensities are multiplied by a factor of 3 . The arrows indicate the frequencies where time-resolved experiments with the same detection setup were performed (Fig. 5). The dotted and dash-dotted lines serve as a guide to the eye.

gated detection. In these experiments, we focus on the dynamics of TO phonons in $a$-nc-Si:H.

In Fig. 4 the low-temperature Stokes and anti-Stokes TO peaks of the $a$-nc-Si:H layer are presented. In these graphs, the different contributions of phonons in $a-\mathrm{Si}: \mathrm{H}$ and nc-Si can be recognized. Remarkably, the part of the anti-Stokes spectrum that corresponds to the TO phonons in the nanocrystals appears to peak at an approximately $10-\mathrm{cm}^{-1}$ lower energy than in the Stokes spectrum. This suggests that during the pulses it is easier to maintain a phonon population at the lower phonon frequencies $\left(\sim 500 \mathrm{~cm}^{-1}\right)$, i.e., closer to the maximum of the amorphous TO peak than at the higher frequencies $\left(\sim 515 \mathrm{~cm}^{-1}\right)$, closer to the center of the crystalline TO peak (at $520 \mathrm{~cm}^{-1}$ ). An obvious explanation for these observations is that phonons at higher frequencies decay faster than at lower frequencies. To verify this interpretation, we employed the pump-probe setup and studied the decay of the anti-Stokes intensity at three selected frequencies within the TO peak: 495,505 , and $515 \mathrm{~cm}^{-1}$, as indicated by the arrows in Fig. 4. Results of these experiments are presented in Fig. 5, which shows the time-dependent part of $I_{\mathrm{AS}}$ measured at the selected frequencies as a function of

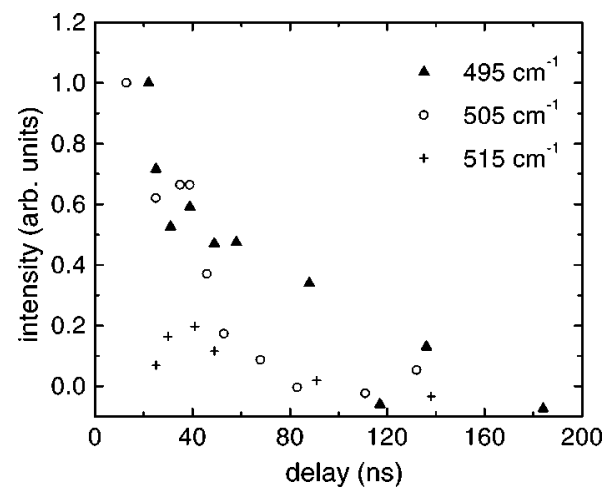

FIG. 5. Normalized time-dependent anti-Stokes intensities measured at three frequencies within the TO peak of $a-\mathrm{nc}-\mathrm{Si}: \mathrm{H}$ as a function of the delay. 
(1)

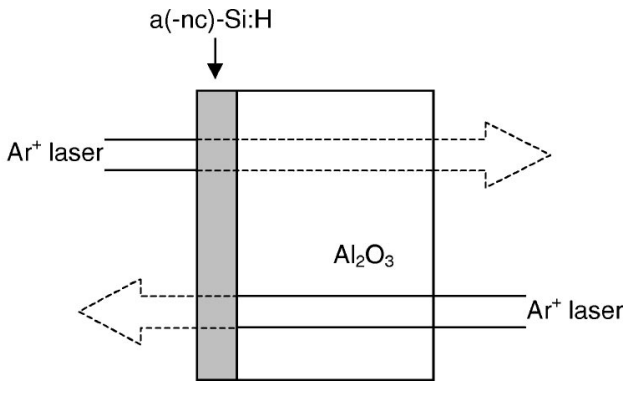

FIG. 6. Schematic representation of the two configurations used for the phonon-induced luminescence experiments.

the delay between pump and probe pulses. Apparently, the decay of phonons at the lowest frequency $\left(495 \mathrm{~cm}^{-1}\right)$ is the slowest ( $\tau \sim 50 \mathrm{~ns})$. For $505 \mathrm{~cm}^{-1}$, the decay is faster, and at a frequency of $515 \mathrm{~cm}^{-1}$, it is too fast $(<10 \mathrm{~ns})$ to be observed with our setup. (Recall that at zero delay a strong contribution is present at $515 \mathrm{~cm}^{-1}$.)

\section{B. Experiments with the ruby phonon detector}

To examine the transport of $29-\mathrm{cm}^{-1}$ phonons through the $a-\mathrm{Si}: \mathrm{H}$ and $a-\mathrm{nc}-\mathrm{Si}: \mathrm{H}$ layers, the experiments with the ruby phonon detector were carried out for two configurations (see Fig. 6): one in which the laser beam was incident at the $a(-\mathrm{nc})$-Si:H side of the sample, and phonons had to travel $\sim 300 \mathrm{~nm}$ from the phonon source to the detector, and a second configuration where the laser entered the sample from the side of the substrate, corresponding to an average distance between the phonon source and detector equal to the penetration depth of the exciting light $(\sim 100 \mathrm{~nm})$. In the following, the two configurations are referred to as configuration (1) and configuration (2), respectively.

Results of the experiments with the ruby phonon detector are collected in Fig. 7. The graphs show the $R_{2}(t) / R_{1}(t)$ intensity induced by the phonons injected from the $a$-nc-Si:H layer, measured for two excitation powers, both for configu-

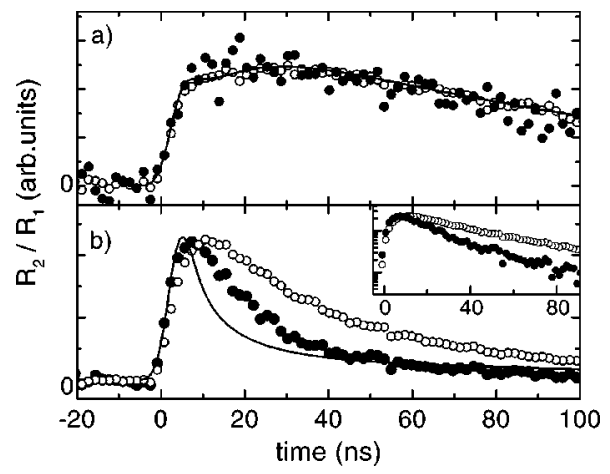

FIG. 7. Normalized phonon-induced signals from the $a$-nc-Si:H layer for two excitation powers: $3.6 \mathrm{~mW}(\bigcirc)$ and $0.9 \mathrm{~mW}(\mathbf{O})$. Graphs in (a) and (b) correspond to the signals obtained for configuration (1) and (2), respectively. For comparison, the baseline levels of the signals obtained in configuration (1) have been subtracted. The inset of graph (b) displays the data collected for configuration (2) on a semilogarithmic plot. The solid lines represent fits based on an elastic diffusion model, as discussed in the text. rations (1) and (2). The temporal shapes of the signals obtained for the two configurations are clearly different. Especially the trailing edge of $R_{2}(t) / R_{1}(t)$ appears to be much longer $(\sim 0.5 \mu \mathrm{s})$ when the phonons have to travel a longer distance from the phonon source to the detector. We note that for the 4-MHz repetition rate at which these measurements were taken, the phonon-induced signals recorded for configuration (1) even do not completely vanish during the interval between two laser pulses, resulting in a nonzero baseline level of $R_{2}(t) / R_{1}(t)$ before $t=0$. In experiments performed at a repetition rate of $800 \mathrm{kHz}$ the interval between two laser pulses turned out to be long enough to let the baseline vanish. To facilitate comparison of the different time traces, in Fig. 7(a), the baseline levels have been subtracted.

As can be seen in Fig. 7(a), the temporal shape of the curve measured for configuration (1) remains virtually the same when the power is decreased by a factor of four. Signals obtained for configuration (2), however, appear to become significantly faster when the power is decreased by the same amount [see Fig. 7(b) and inset]. Both the time scales of the rise and the decay of $R_{2}(t) / R_{1}(t)$ are shorter for lower power. The integrated $R_{2}(t) / R_{1}(t)$ intensity, however, increases linearly with excitation power for both configurations. This suggests that no $29-\mathrm{cm}^{-1}$ phonons get lost due to the high excitation level during their travel through the $a$-nc-Si:H film.

Different than for the $a$-nc-Si:H sample, in the case of $a-\mathrm{Si}: \mathrm{H}$, results of the measurements performed for configuration (1) resemble those for configuration (2) for each of the excitation powers. In this material, $R_{2} / R_{1}$ exhibits a steeper rise for lower powers. On the other hand, the trailing edge decays on the same $\sim 50$-ns time scale for different excitation powers, i.e., much faster than the decay of the $R_{2} / R_{1}$ signals presented above for $a$-nc-Si:H for configuration (1). These observations are in agreement with the results reported for $a$-Si:H by Scholten et al. ${ }^{11}$ and are therefore not shown.

\section{DISCUSSION}

The results of the pulsed Raman and phonon transport experiments obtained for $a$-nc-Si:H show that long-lived vibrational signals can be measured, just like in $a-\mathrm{Si}: \mathrm{H}$ containing no crystallites. As will be discussed below, however, the dynamics of phonons in $a$-nc-Si:H is in several aspects distinctly different from both the phonon dynamics in $a-\mathrm{Si}: \mathrm{H}$ and in $c$-Si, indicating the strong influence of the materials microstructure on the dynamical vibrational properties.

\section{A. Raman experiments}

As already noted, the quadratic dependence of the antiStokes intensity and linear dependence of the Stokes intensity on excitation power demonstrate that intense optical excitation of $a$-nc-Si:H leads to the creation of a nonequilibrium phonon population, as is the case in $a-\mathrm{Si}: \mathrm{H}$. The decay of this population can be investigated via the temporal evolution of the anti-Stokes spectra. Thus, we found that the phonon signals decay on time scales of $50-70 \mathrm{~ns}$ for almost 
the entire range of frequencies addressed by the anti-Stokes Raman experiments (see Fig. 3). The values of these decay times are very close to the values found for the lifetimes of LA- and TO-like vibrations in $a-\mathrm{Si}(: \mathrm{H})$, and are extremely long compared to the 10-ps lifetime of TO phonons in $c-\mathrm{Si}^{18}$ In case this longevity is related to the materials microstructure, in particular the differences in the behavior observed for phonons in $a$-nc-Si:H and $a$-Si:H may give indications for possible explanations. The most pronounced differences between the results of the Raman experiments in $a-\mathrm{nc}-\mathrm{Si}: \mathrm{H}$ and $a-\mathrm{Si}: \mathrm{H}$ are that the lifetime of the TO phonons in $a-\mathrm{nc}-\mathrm{Si}: \mathrm{H}$ is frequency dependent, and that TA-like phonons in $a$-nc-Si:H appear to survive over much longer periods than in $a-\mathrm{Si}: \mathrm{H}$. These points will be addressed in the remainder of this section.

The time-resolved anti-Stokes Raman experiments of Fig. 5 demonstrate that in $a$-nc-Si:H the decay rate of the TO phonons is frequency dependent. The phonon lifetimes increase with decreasing frequency, from less than $10 \mathrm{~ns}$ at $515 \mathrm{~cm}^{-1}$ to $\sim 50 \mathrm{~ns}$ at $495 \mathrm{~cm}^{-1}$. This effect may be related to the polydispersity of the nc-Si particles in the $a$-nc-Si:H film. Smaller particles give rise to TO peaks at lower frequencies $\omega_{\mathrm{nc}},{ }^{2,3}$ so that the frequency dependence of the TO lifetime may in fact be a size dependence. According to this interpretation, the difference in Stokes and antiStokes TO peak frequencies that showed up in Fig. 4 indicates that during one laser pulse, the larger particles $(d$ $\gtrsim 4.5 \mathrm{~nm})$ that correspond to the larger Raman shifts $\left(\omega_{\mathrm{nc}}\right.$ $\gtrsim 515 \mathrm{~cm}^{-1}$ ) become less populated with TO phonons than small nanocrystals $(\sim 1.5 \mathrm{~nm})$ that give the smaller TO Raman shifts $\left(\omega_{\mathrm{nc}} \sim 495 \mathrm{~cm}^{-1}\right)$. In that case, only the small crystallites produce the nonequilibrium anti-Stokes TO intensity, whereas all particles contribute to the spontaneous Stokes signals. That would suggest that the anharmonic decay of TO vibrations is suppressed in the smaller particles. ${ }^{19}$

We propose that the slowing down of the anharmonic decay is related to phonon confinement effects, which become important as soon as the characteristic length scale, say, the wavelength of $\mathrm{THz}$ phonons, approaches the size of the nanocrystals. In this connection, we note that in the temperature region that corresponds to the frequency of $\mathrm{THz}$ phonons, one has reported an excess of the phonon specific heat of lead and palladium nanoparticles of similar sizes as ours, compared to that of their bulk-crystalline counterparts. ${ }^{20,21}$ This behavior has been attributed to phonon confinement effects. ${ }^{22}$

An important difference between bulk crystalline materials on the one hand and nanocrystals on the other is the discreteness of the spectrum that exists in an isolated nanocrystallite. ${ }^{23}$ Directly connected with that is the existence of a minimum frequency, $\omega_{\min }$, for the acousticphonon branch in an isolated nanocrystal. Inherent to the existence of an $\omega_{\min }$ is also the occurrence of a gap between the ground state and the first vibrational level. Consequently, physical properties that are determined by the vibrational density of states, like the phonon specific heat and the anharmonic decay, can deviate significantly from those of bulk materials. Depending on the actual spectrum of eigenstates in a specific energy range, the physical properties can be sup- pressed or enhanced compared to the bulk crystalline case. For example, the phonon specific heat is expected to fall exponentially below a temperature that corresponds to the energy of the gap between the ground state and first vibrational level. ${ }^{21}$

In the following we explore if the concept of a discrete vibrational spectrum may explain a size dependence of the anharmonic lifetime of the TO vibrations in nc-Si. For this purpose, we need to know the eigenfrequency spectrum of the nc-Si crystallites. The vibrational eigenfrequencies of a homogeneous spherical body were calculated by Lamb in $1882 .{ }^{24}$ To obtain a measure of the vibrational spectrum of $\mathrm{nc}-\mathrm{Si}$ for the relevant frequencies we follow a procedure based on the work of Lamb, which has been outlined for other nanocrystalline materials by various authors. ${ }^{22,25}$ To make the problem tractable, the nanocrystals are in this approach represented by homogeneous spheres that are considered as isotropic continuous elastic bodies. Then, the eigenfrequencies of a particle are determined by the solutions of the wave equation $\left(\nabla^{2}+q^{2}\right) \vec{u}=0$, with the stress-free $(\vec{\nabla} \cdot \vec{u}=0)$ or, alternatively, displacement free $(u=0)$ boundary conditions on the surface of the particle, for a free and clamped sphere, respectively. The eigenvectors $q_{l m}$ can be found and are given by $q_{l m}=a_{l m} /(d / 2)$, with $d$ the particle diameter. For a free sphere, $a_{l m}$ is the $m$ th zero of the derivative of the $l$ th spherical Bessel function of the first kind. In the case of a clamped surface, $a_{l m}$ represents the $m$ th zero of the $l$ th Bessel function itself. The degeneracy of $q_{l m}$ is $(2 l+1)$. For the eigenfrequencies $\omega_{l m}$ we simply take $\omega_{l m}$ $=c q_{l m}$, where $c$ is the average sound velocity calculated from the transverse and longitudinal sound velocities (for $\mathrm{Si}$, $c \approx 6.3 \times 10^{3} \mathrm{~ms}^{-1}$ ).

Probably, most particles in our $a$-nc-Si:H sample are neither completely free, nor fully clamped, and it is unlikely that the crystallites are perfect spheres. Also the validity of the elastic continuum approximation is questionable, especially for particles with diameters only several times the atomic distance of Si. However, we believe that spectra calculated as described above give a rough, but faithful, measure for the dependence of $\omega_{\min }$ and the spacing between the modes, on the size of the Si particles. In fact, in Raman experiments on nucleated glasses a remarkably good agreement of the calculated and measured size dependence of $\omega_{\text {min }}$ has been observed for crystallites with diameters between 10 and $40 \mathrm{~nm} \cdot{ }^{26}$

We expect that the discreteness of the vibrational spectra of nanostructured materials has effects on the phonon dynamics. If the modes that are required for the regular anharmonic break up of a phonon into two lower frequency modes are not available, the bulk-crystalline decay channels are suppressed, which decreases the decay rate. The phonon experiments on porous corundum indeed showed that the modes just above $\omega_{\min }$ have extremely long lifetimes. ${ }^{5,6}$ The TO modes examined in our experiments have a frequency that is several times higher than the $\omega_{\text {min }}$ of nc-Si particles with diameters between 1.5 and $5 \mathrm{~nm}$. Hence, a sizedependent decay by anharmonic break up is more probably 


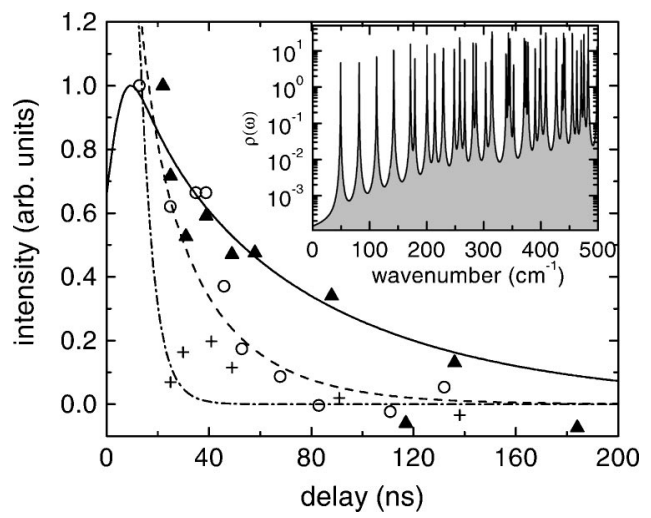

FIG. 8. Decay of the Raman signals calculated using Eq. (1) for $d=1.5 \mathrm{~nm}$ (solid line), $d=2.5 \mathrm{~nm}$ (dashed line), and $d=4.5 \mathrm{~nm}$ (dash-dotted line), compared with the experimental results for $495 \mathrm{~cm}^{-1}(\boldsymbol{\Delta}), \quad 505 \mathrm{~cm}^{-1}(\bigcirc)$, and $515 \mathrm{~cm}^{-1}(+)$. The inset shows the vibrational density of states per unit of volume for $d$ $=2.5 \mathrm{~nm}$. The Lorentzians have a width of $0.1 \mathrm{~cm}^{-1}$.

related to the relative mode spacing of the accepting phonon modes than to the exact value of $\omega_{\min }$.

In crystalline silicon, the $\vec{k}=\overrightarrow{0}$ TO phonons that are observed in a Raman experiment decay into two acoustic phonons of half the TO frequency, at a rate that scales with the square of the density of final states per unit of volume. ${ }^{27}$ In analogy with that, we assume that also in a crystallite the rate of anharmonic break up of a TO vibration of frequency $\omega_{\text {TO }}$ is determined by the density of acoustic states per unit of volume at frequency $\left(\omega_{\mathrm{TO}} / 2\right), \quad \rho\left(\omega_{\mathrm{TO}} / 2\right)$, fulfilling both energy conservation and phase-matching conditions. Then, the intensity $I(t)$ of the TO Raman peak of a particular size class of nanoparticles that support TO phonons of the given frequency decays in time according to

$$
I(t) \propto g(t) *\left\langle\exp \left[-\left(\frac{\rho\left(\omega_{\mathrm{TO}} / 2\right)}{\rho_{0}}\right)^{2} \frac{t}{\tau_{0}}\right]\right\rangle .
$$

Here $\rho_{0}$ is the crystalline density of states at frequency $\left(\omega_{\mathrm{TO}} / 2\right), \tau_{0}$ is the lifetime of TO phonons in $c-\mathrm{Si}(10 \mathrm{ps})$, the average is taken over the nanoparticles in that class, and the convolution is taken with the experimental profile $g(t)$.

To make the problem tractable, we take the eigenfrequency spectra calculated above for free spheres of diameter $d$ as a measure of the density of acoustic modes in nc-Si particles of corresponding size. As the vibrational excitations have a finite lifetime, the resonances are expected to have a Lorentzian line shape with a finite width. For simplicity, the same width is assigned to all Lorentzians. The inset of Fig. 8 shows an example of $\rho(\omega)$ computed in such a way for 2.5-nm particles, and a Lorentzian linewidth of $0.1 \mathrm{~cm}^{-1}$.

To further evaluate Eq. (1), we substitute $\rho(\omega)$ for $\rho\left(\omega_{\mathrm{TO}} / 2\right)$ and replace the averaging over the class of nanoparticles by a frequency averaging around $\left(\omega_{\mathrm{TO}} / 2\right)$ over a range corresponding with the typical mode distance for particles of the class under investigation (e.g., $\sim 20 \mathrm{~cm}^{-1}$ for 2.5-nm particles, see the inset of Fig. 8). Finally, we take a Gaussian line shape with full width at half maximum (FWHM) of 15 ns for the instrumental profile. In Fig. 8 we plot $I(t)$ for three classes of nanoparticles, with diameters of $1.5,2.5$, and $4.5 \mathrm{~nm}$, and compare the decay with the experimental results of Fig. 5. The only adjustable parameter in the calculation is the natural linewidth of the modes. We find that $I(t)$ qualitatively tracks our experimental findings for a natural linewidth of $0.1 \mathrm{~cm}^{-1}$.

The analysis presented here suggests that the frequency dependence of the TO lifetimes can be explained as resulting from a size-dependent suppression of the anharmonic TO decay. We emphasize, however, that this is only true if the modes to which the TO phonons decay are narrow enough in frequency $\left(\sim 0.1 \mathrm{~cm}^{-1}\right)$. In other words, the acoustic resonators have to be sufficiently decoupled from their surroundings: their quality factor has to be high enough. For a resonator with a mode distance of $20 \mathrm{~cm}^{-1}$ and linewidth of the resonances of $0.1 \mathrm{~cm}^{-1}$, the $Q$ factor is $200 .^{28}$ Obviously, only a systematic study of materials with different crystallite sizes would provide conclusive evidence for the above predicted size dependence of the TO decay time. In this respect, we note that we have only been able to perform preliminary experiments on one other $a$-nc-Si:H sample containing larger crystallites. Results of those measurements confirm a faster TO decay for larger crystallite sizes. We hope that a systematic study will be performed in the future.

The components of the Raman spectra that are assigned to the nc-Si particles are in frequency exactly in the region between the TO peak of $c$-Si and that of $a-\mathrm{Si}: \mathrm{H}$. The measured lifetimes of the modes in this region are also in between the values found for TO modes in $c$-Si and $a-\mathrm{Si}: \mathrm{H}$, decreasing from $\tau<10 \mathrm{~ns}$ for the frequencies close to the $c$-Si resonance to $\tau \sim 50 \mathrm{~ns}$ for frequencies approaching the TO peak of $a$-Si. The reason for the long decay times of phonons in $a-\mathrm{Si}: \mathrm{H}$ without nanocrystallites is still unexplained. However, if we associate the observed longevity with the above interpretation of the long lifetimes measured in $a$-nc-Si:H, one is tempted to consider $a$-Si:H as a material containing a large number of small $(\sim 1 \mathrm{~nm})$ isolated regions, in which the positions of the atoms are correlated. Indeed, one finds that $a$-Si:H exhibits "medium" range order on nanometer length scales. ${ }^{14}$ If, in addition, vibrations in neighboring regions are sufficiently decoupled, the vibrational spectrum becomes discrete, resulting in a suppressed decay of the TO modes.

Also consistent with this interpretation is the observation (Fig. 3) that the vibrations in the spectral region of the TA phonons decay much slower in $a$-nc-Si:H than in $a-\mathrm{Si}: \mathrm{H}$. As phonons of these frequencies are not Raman active in $c-\mathrm{Si}$, the signals we detect in $a$-nc-Si:H are most probably caused by vibrations residing in the $a-\mathrm{Si}: \mathrm{H}$ regions of the material. Considering the amorphous tissue in between the crystallites, we note once more that also the $a-\mathrm{Si}: \mathrm{H}$ tissue is structured on length scales of the order of $10 \mathrm{~nm}$ due to the presence of the crystallites. This may in turn suppress the anharmonic break up of TA vibrations in the amorphous material in the same way as the decay of the TO modes is suppressed in the $a$-Si:H samples without crystallites. The structure on a larger length scale affects the dynamics of vibrations of lower fre- 
quencies. Pure $a-\mathrm{Si}: \mathrm{H}$ is not structured on $10-\mathrm{nm}$ length scales, ${ }^{29}$ consistent with the observed short lifetimes of the TA modes in that material.

\section{B. Long-lasting phonon-induced luminescence signals}

In the experiments performed with the ruby phonon detector, the transport was investigated of phonons of a much lower frequency $\left(29 \mathrm{~cm}^{-1}\right)$ than of the phonons addressed in the Raman measurements. Also in these experiments we found that the phonon dynamics in $a-\mathrm{nc}-\mathrm{Si}: \mathrm{H}$ is markedly different from that in $a-\mathrm{Si}: \mathrm{H}$. The outcome of the transport experiments on $a$-Si:H is consistent with the results published by Scholten et al., who extensively studied the scattering of $29-\mathrm{cm}^{-1}$ phonons in $a-\mathrm{Si}: \mathrm{H} .{ }^{11}$ They showed that in $a-\mathrm{Si}: \mathrm{H}$ the transport of $29-\mathrm{cm}^{-1}$ phonons is governed by elastic spatial diffusion with a diffusion coefficient of $\sim 1 \mathrm{~cm}^{2} / \mathrm{s}$. Encouraged by their analysis, we try to explain the results of the phonon transport measurements on $a$-nc-Si:H in terms of an elastic diffusion model.

The $29-\mathrm{cm}^{-1}$ phonons were generated in the $a-\mathrm{nc}-\mathrm{Si}: \mathrm{H}$ film by means of optical excitation. Due to the finite penetration depth of the green light into the sample, not all phonons are created at the same depth, and consequently do not have to travel the same distance to the ruby detector. Below, we first solve the diffusion equation for a phonon density $n\left(x, x^{\prime}, t\right)$, created at $t=0$ at a depth $x^{\prime}>0$ in an $a$-nc-Si:H layer. Subsequently, a solution is constructed for the situation where phonons are generated according to a specified excitation profile with a certain depth into the sample. From that, the total flux $\Phi(t)$ of phonons injected into the $\mathrm{Al}_{2} \mathrm{O}_{3}$ that are resonant with the ruby detector can be determined. The results for $\Phi(t)$ will be compared with the measured temporal evolution of the phonon induced luminescence intensity.

In the case where the transport of $29-\mathrm{cm}^{-1}$ phonons through the $a$-nc-Si:H layer is entirely determined by elastic spatial diffusion and characterized by a single diffusion coefficient $D$, the one-dimensional diffusion equation for $n\left(x, x^{\prime}, t\right)$ applies, which reads

$$
\frac{\partial n\left(x, x^{\prime}, t\right)}{\partial t}=D \frac{\partial^{2} n\left(x, x^{\prime}, t\right)}{\partial x^{2}} .
$$

The initial condition is $n\left(x, x^{\prime}, 0\right)=n_{0} \delta\left(x-x^{\prime}\right)$, with $n_{0}$ the number of resonant phonons injected per unit area, and $\delta(x$ $\left.-x^{\prime}\right)$ the Dirac $\delta$ function. The position $x=0$ corresponds to the interface between the $a$-nc-Si:H layer and the surrounding He gas. For an infinite medium, the solution of Eq. (2) is given by

$$
n\left(x, x^{\prime}, t\right)=\frac{n_{0}}{\sqrt{4 \pi D t}} \exp \left[-\frac{\left(x-x^{\prime}\right)^{2}}{4 D t}\right],
$$

which corresponds to a phonon flux $\phi\left(x, x^{\prime}, t\right)=-\partial n / \partial x$ of

$$
\phi\left(x, x^{\prime}, t\right)=\frac{2 \pi n_{0}\left(x-x^{\prime}\right)}{(4 \pi D t)^{3 / 2}} \exp \left[-\frac{\left(x-x^{\prime}\right)^{2}}{4 D t}\right] .
$$

Boundary conditions are imposed by the properties of the interfaces between the sample and the substrate at one side, and between the sample and the $\mathrm{He}$ gas at the other side. The $\mathrm{Al}_{2} \mathrm{O}_{3}$ substrate is assumed to act as an ideal sink for the $29-\mathrm{cm}^{-1}$ phonons, while the He surface is taken $100 \%$ reflective. Because of the first condition, the phonon density at the interface with the substrate $(x=L)$ should always vanish: $n\left(L, x^{\prime}, t\right)=0$. According to the second condition, the net flux of $29-\mathrm{cm}^{-1}$ phonons through the He surface has to vanish at all times. The problem is solved using the method of images. ${ }^{11}$ To fulfill both boundary conditions, the initial distribution $n\left(x, x^{\prime}, 0\right)$ has to be symmetric around $x=0$, and antisymmetric around $x=L$. This is accomplished by introducing a positive $\delta$ peak $n_{0} \delta\left(x+x^{\prime}\right)$ at $x=-x^{\prime}$, and two negative peaks at $x=2 L-x^{\prime}$ and $x=2 L+x^{\prime}$. To preserve symmetry around $x=0$ again two $\delta$ peaks have to be added at $x=-2 L+x^{\prime}$ and $x=-2 L-x^{\prime}$, destroying again the antisymmetry around $x=L$. To satisfy the two conditions, the procedure has to be repeated to infinity, leading to an infinite number of image phonon distributions. The resulting phonon flux $\widetilde{\Phi}\left(x^{\prime}, t\right)$ at the interface with the $\mathrm{Al}_{2} \mathrm{O}_{3}$ substrate is equal to

$$
\begin{aligned}
\widetilde{\Phi}\left(x^{\prime}, t\right)= & \sum_{i=0}^{\infty}(-1)^{i} \frac{4 \pi n_{0}}{(4 \pi D t)^{3 / 2}}\left\{\left[(2 i+1) L-x^{\prime}\right]\right. \\
& \times \exp \left[-\frac{\left((2 i+1) L-x^{\prime}\right)^{2}}{4 D t}\right]+\left[(2 i+1) L+x^{\prime}\right] \\
& \left.\times \exp \left[-\frac{\left((2 i+1) L+x^{\prime}\right)^{2}}{4 D t}\right]\right\} .
\end{aligned}
$$

If the phonons are created according to an exponentially decaying excitation profile, with a penetration depth $1 / \alpha$ into the sample, the total phonon flux $\Phi_{1}(t)$ for configuration (1) at $x=L$ is described by

$$
\Phi_{1}(t) \propto \int_{0}^{L} d x^{\prime} e^{-\alpha x^{\prime}} \tilde{\Phi}\left(x^{\prime}, t\right) .
$$

To calculate the phonon flux $\Phi_{2}(t)$ for configuration (2), $\exp \left[-\alpha x^{\prime}\right]$ in Eq. (6) is replaced by $\exp \left[-\alpha\left(L-x^{\prime}\right)\right]$. When comparing $\Phi_{1,2}(t)$ with the $R_{2} / R_{1}$ obtained from the measurements, the 10-ns resolution of the ruby phonon detector has to be taken into account. This is done by convoluting $\Phi_{1,2}(t)$ with a Gaussian of the corresponding width. Then $\Phi_{1,2}$ can be fitted to the data, substituting $L=0.4 \mu \mathrm{m}$, and using $1 / \alpha$ and $D$ as fitting parameters.

By fitting $\Phi_{1}(t)$ to the data acquired in configuration (1), we obtained for the values of the parameters $1 / \alpha=150$ $\pm 20 \mathrm{~nm}$, and $D=(6 \pm 0.5) \times 10^{-3} \mathrm{~cm}^{2} / \mathrm{s}$. A penetration depth of $150 \mathrm{~nm}$ seems quite reasonable for 514-nm photons traversing $a$-nc-Si:H. Note, however, that the value for $D$ is more than two orders of magnitude smaller than that determined for $29-\mathrm{cm}^{-1}$ phonons in $a-\mathrm{Si}: \mathrm{H}$ by Scholten et al. ${ }^{11}$ As can be seen from Fig. 7(a), $\Phi_{1}(t)$ describes the data quite well for these values of $1 / \alpha$ and $D$. However, Fig. 7(b) shows that $\Phi_{2}(t)$ by no means fits the experimental traces for configuration (2), for the same values of $1 / \alpha$ and $D$. The 
observed temporal profile is significantly slower than predicted by the above model. We note that our model with a constant $D$ cannot explain the excitation power dependence of the traces recorded for this configuration.

Scholten et al. also observed power dependences of the phonon signals in optically excited $a-\mathrm{Si}: \mathrm{H}$. They demonstrated that incorporation of inelastic scattering of $29-\mathrm{cm}^{-1}$ phonons off higher-frequency phonons made the diffusion model to account for the results of the experiments. For higher excitation powers, more high-frequency phonons are generated, resulting in a higher scattering rate of the $29-\mathrm{cm}^{-1}$ phonons and a deceleration of the rise of the $R_{2} / R_{1}$ signals, as they observed. Further, in their case, the decay of the $R_{2} / R_{1}$ intensity was shown to be independent of excitation power, and characterized by a 45 -ns decay time. Scholten et al. related this tail to the generation of $29-\mathrm{cm}^{-1}$ phonons by the anharmonic break up of long-lived higherfrequency phonons.

We note that the results of our experiments on $a$-nc-Si:H are markedly different from those reported by Scholten et al. for $a-\mathrm{Si}: \mathrm{H}$. First of all, the diffusion of $29-\mathrm{cm}^{-1}$ phonons in $a$-nc-Si:H is much slower than in $a-\mathrm{Si}: \mathrm{H}$. Further, in $a$-nc-Si:H, in contrast to $a$-Si:H, the decay of the $R_{2} / R_{1}$ signals in configuration (2) clearly slows down if the excitation power is increased. This observation, and the difference in decay times of $R_{2} / R_{1}$ measured for configurations (1) and (2), suggests that in $a$-nc-Si:H the tail of the $R_{2} / R_{1}$ signals is not characterized by the decay of the high-frequency vibrations. On the other hand, an increase of the scattering rate for higher phonon occupation numbers would be consistent with the power dependence we observed in $a$-nc-Si:H for $R_{2} / R_{1}$ in configuration (2). Such effects are expected to be less pronounced in configuration (1). In configuration (2), namely, the $29-\mathrm{cm}^{-1}$ phonons have to traverse the highest excited part of the material before reaching the phonon detector, whereas they travel through lower excited regions in configuration (1). This also explains why the value of $D$ obtained from the results for configuration (1) corresponds in configuration (2) to a temporal profile that is significantly faster than the observed traces.

Apart from the power-dependent effects, the results for configuration (1) demonstrate that the diffusion of $29-\mathrm{cm}^{-1}$ phonons through relatively low excited material is much slower in $a$-nc-Si:H than in $a$-Si:H. In this respect, we recall that the $a$-nc-Si:H material is structured on length scales $(\sim 5 \mathrm{~nm})$ comparable to the wavelength of the phonons under investigation. This can lead to strong elastic scattering, resulting in slow diffusion. ${ }^{5,6}$ Regions may even exist that cannot support the $29-\mathrm{cm}^{-1}$ phonons at all, further obstructing propagation of such vibrations. Indicative of anomalously slow diffusion is that for acoustic phonons of the average sound velocity, the classical mean free path corresponding to a diffusion constant of $6 \times 10^{-3} \mathrm{~cm}^{2} / \mathrm{s}$ would be $0.3 \mathrm{~nm}$, which would be much smaller than their wavelength.

We conclude that the transport of $29-\mathrm{cm}^{-1}$ phonons in $a$-nc-Si:H is strongly influenced by the structure of the material. To draw any firm conclusions about the processes responsible for the low, power-dependent diffusion coefficient, more experiments are required. For example, measurements as a function of the thickness of the $a$-nc-Si:H layer, or for different phonon frequencies, are expected to provide valuable information.

The results of the phonon-induced lumincescence experiments confirm that the presence of $\sim 5$-nm sized structures in $a$-nc-Si:H slows down the dynamics of $29-\mathrm{cm}^{-1}$ phonons. Similar effects may occur for higher-frequency vibrations in $a-\mathrm{Si}: \mathrm{H}$ if $a-\mathrm{Si}: \mathrm{H}$ is structured on a nanometer lengthscale.

\section{SUMMARY AND CONCLUSIONS}

In summary, we report on the results of pulsed Raman and phonon-induced luminescence experiments on a mixed amorphous-nanocrystalline $\mathrm{Si}$ system $(a$-nc-Si:H). The techniques were used to study the dynamics of terahertz phonons, and compare it with the behavior of phonons in $a-\mathrm{Si}: \mathrm{H}$. The main goal of the experiments was to investigate the influence of the presence of nanometer scale structures on the phonon dynamics.

From the Raman experiments, we find that the decay time of phonons in the spectral region of the TO vibrations in the crystallites is frequency dependent. The lifetimes are observed to increase with decreasing frequency, from less than $10 \mathrm{~ns}$ at $515 \mathrm{~cm}^{-1}$ to $\sim 30 \mathrm{~ns}$ at $505 \mathrm{~cm}^{-1}$. We present a model to explain the frequency-dependent TO lifetimes as resulting from a size-dependent suppression of the anharmonic decay inside the nanoparticles: the smaller the particles are, the slower the anharmonic decay of the TO modes. A crucial ingredient of our model is that the acoustic resonances (Lamb modes) of the nanoparticles have a narrow natural linewidth $\left(\sim 0.1 \mathrm{~cm}^{-1}\right)$. We go even further and connect this typical behavior in nanocrystalline silicon with the long TO lifetimes measured in $a-\mathrm{Si}: \mathrm{H}$, and we suggest that one may consider $a-\mathrm{Si}: \mathrm{H}$ as a material containing a large amount of small $(\sim 1 \mathrm{~nm})$ decoupled regions, in which the decay of the TO phonons can be severely suppressed.

Another conclusion that follows from the pulsed Raman measurements is that in $a$-nc-Si:H, the modes in the spectral region of the TA phonons decay much slower than in $a-\mathrm{Si}: \mathrm{H}$. The explanation for the longevity of the TO phonons also accounts for this observation. Compared to the length scale of the structures that are of importance for the dynamics of the TO phonons, structures of larger dimensions appear to influence the dynamics of the lower-frequency TA vibrations.

Also the outcome of the phonon transport measurements illustrates the effect of nanometer scale structure on the behavior of $\mathrm{THz}$ phonons. The results are described in terms of elastic spatial diffusion, characterized by a diffusion coefficient $D \sim(6 \pm 0.5) \times 10^{-3} \mathrm{~cm}^{2} / \mathrm{s}$, a value that is more than two orders of magnitude lower than for $29-\mathrm{cm}^{-1}$ phonons in $a$-Si:H. The slow diffusion is ascribed to the anomalously strong scattering of phonons inside a material that is structured on length scales equal to the wavelength of the phonons. The diffusion of $29-\mathrm{cm}^{-1}$ phonons is observed to become even slower when more vibrations are excited, and an as yet unexplained additional scattering mechanism comes into play. 


\section{ACKNOWLEDGMENTS}

We acknowledge F.J.M. Wollenberg, P. Jurrius, and C.R. de Kok for invaluable technical assistence. Of the A.F. Ioffe Physical-Technical Institute in St. Petersburg we thank N.A. Feoktistov for providing us with the $a-\mathrm{Si}: \mathrm{H}$ and $a$-nc-Si:H samples, and A.A. Kaplyanskii for fruitful discussions. This work is part of the research program of the Dutch foundation "Stichting voor Fundamenteel Onderzoek der Materie (FOM)," which is financially supported by the "Nederlandse organisatie voor Wetenschappelijk Onderzoek (NWO).', We also acknowledge the Russian Foundation for Basic Research for financial support.
${ }^{1}$ Phonons in Semiconductor Nanostructures, edited by J. P. Leburton, J. Pasqual, and C. Sotomayor Torres, Vol. 236 of Nato Science Series E: Applied Sciences (Kluwer Academic, Dordrecht, 1993).

${ }^{2}$ H. Richter, Z. P. Wang, and L. Ley, Solid State Commun. 39, 625 (1981).

${ }^{3}$ I. H. Campbell and P. M. Fauchet, Solid State Commun. 58, 739 (1986); Z. Iqbal and S. Veprek, J. Phys. C 15, 377 (1993).

${ }^{4}$ B. Champagnon, B. Andriasolo, and E. Duval, J. Chem. Phys. 94, 5237 (1991); A. Tanaka, S. Onari, and T. Arai, Phys. Rev. B 47, 1237 (1993); M. Fujii and Y. Kanzawa, ibid. 54, R8373 (1996).

${ }^{5}$ S. P. Feofilov, A. A. Kaplyanskii, and R. I. Zakharchenya, J. Lumin. 66\&67, 349 (1996); A. A. Kaplyanskii, S. P. Feofilov, and R. I. Zakharchenya, Opt. Spektrosk. 79, 709 (1995) [Opt. Spectrosc. 79, 653 (1995)].

${ }^{6}$ S. P. Feofilov, A. A. Kaplyanskii, A. B. Kulinkin, and R. I. Zakharchenya, Physica B 263-264, 695 (1999).

${ }^{7}$ S. P. Feofilov, A. A. Kaplyanskii, and M. B. Melnikov, Physica B 219-220, 773 (1996).

${ }^{8}$ A. J. Scholten, A. V. Akimov, and J. I. Dijkhuis, Phys. Rev. B 47, 13910 (1993); A. J. Scholten, P. A. W. E. Verleg, J. I. Dijkhuis, A. V. Akimov, R. S. Meltzer, and R. Orbach, Solid State Phenom. 44-46, 289 (1995).

${ }^{9}$ S. A. Basun, P. Deren, S. P. Feofilov, A. A. Kaplyanskii, and W. Strek, in Phonons '89, edited by S. Hunklinger, W. Ludwig, and G. Weiss (World Scientific, Singapore, 1990), Vol. 2, p. 1424.

${ }^{10}$ A. J. Scholten and J. I. Dijkhuis, Phys. Rev. B 53, 3837 (1996).

${ }^{11}$ A. J. Scholten, A. V. Akimov, and J. I. Dijkhuis, Phys. Rev. B 54, 12151 (1996).

${ }^{12}$ J. Fabian and P. B. Allen, Phys. Rev. Lett. 77, 3839 (1996); S. R. Bickham and J. L. Feldman, Phys. Rev. B 57, 12234 (1998); P. B. Allen, J. L. Feldman, J. Fabian, and F. Wooten, Philos. Mag. B 79, 1715 (1999).

${ }^{13}$ M. H. Brodsky, R. S. Title, K. Weiser, and G. D. Petit, Phys. Rev. B 1, 2632 (1970).

${ }^{14}$ M. M. J. Treacy, J. M. Gibson, and P. J. Keblinski, J. Non-Cryst. Solids 231, 99 (1998); M. M. J. Treacy, P. M. Voyles, and J. M. Gibson, ibid. 266-269, 150 (2000); J. M. Gibson and M. M. J. Treacy, Phys. Rev. Lett. 78, 1074 (1997).

${ }^{15}$ V. G. Golubev, V. Yu Davydov, A. V. Medvedev, A. B. Pevtsov, and N. A. Feoktistov, Fiz. Tverd. Tela (St. Petersburg) 39, 1348 (1997) [Phys. Solid State 39, 1197 (1997)].

${ }^{16}$ K. F. Renk and J. Deisenhofer, Phys. Rev. Lett. 26, 764 (1971).

${ }^{17}$ M. van der Voort, A. V. Akimov, and J. I. Dijkhuis, Phys. Rev. B 62, 8072 (2000); M. van der Voort, G. D. J. Smit, A. V. Akimov, and J. I. Dijkhuis, Physica B 263-264, 283 (1999).

${ }^{18}$ J. Menendez and M. Cardona, Phys. Rev. B 29, 2051 (1984).

${ }^{19}$ Note that experiments presented elsewhere (Ref. 2) show a decrease of the width of the TO peak with increasing crystallite size. In case those linewidths are interpreted as decay rates, that dependence is different from the decrease of the phonon lifetime with increasing crystallite size that we propose. In the following, we explain this different dependency by taking into account the discreteness of the phonon density of states of small isolated nanocrystals. For particle sizes as in Ref. 2, our discrete phonon density of states converges to the continuous density of states of $c$-Si as used in Ref. 2.

${ }^{20}$ V. Novotny and P. P. M. Meincke, Phys. Rev. B 8, 4186 (1975); V. Novotny, P. P. M. Meincke, and J. H. P. Watson, Phys. Rev. Lett. 28, 901 (1972); G. H. Comsa, D. Heitkamp, and H. S. Räde, Solid State Commun. 24, 547 (1977).

${ }^{21}$ F. Bartolomé, J. Bartolomé, C. Benelli, A. Caneschi, D. Gatteschi, C. Paulsen, M. G. Pini, A. Rettori, R. Sessoli, and Y. Volokitin, Phys. Rev. Lett. 99, 382 (1996).

${ }^{22}$ H. P. Baltes and E. R. Hilf, Solid State Commun. 12, 369 (1973).

${ }^{23}$ A. Tamura, K. Higeta, and T. Ichinokawa, J. Phys. C 15, 4975 (1982).

${ }^{24}$ H. Lamb, Proc. London Math. Soc. 13, 189 (1882).

${ }^{25}$ R. Lautenschläger, Solid State Commun. 16, 1331 (1975).

${ }^{26}$ E. Duval, A. Boukenter, and B. Champagnon, Phys. Rev. Lett. 56, 2052 (1986).

${ }^{27}$ P. G. Klemens, Phys. Rev. 148, 845 (1966); R. Orbach, Phys. Rev. Lett. 16, 15 (1966).

${ }^{28}$ Note that in the optical regime $Q$ factors of $\sim 10^{7}$ are found for silica microspheres [see, for instance, M. Cai, O. Painter, and K. J. Vahala, Phys. Rev. Lett. 85, 74 (2000)], indicating that a $Q$ factor of 200 or larger in the acoustic regime is not unreasonable.

${ }^{29}$ R. E. I. Schropp and M. Zeman, Amorphous and Microcrystalline Silicon Solar Cells: Modeling, Materials and Device Technology (Kluwer Academic Publishers, Boston, 1998). 